\title{
The LUMBA UVES stellar parameter pipeline
}

\author{
Alvin Gavel ${ }^{1}$, Pieter Gruyters ${ }^{1}$, Ulrike Heiter ${ }^{1}$, Andreas J. Korn ${ }^{1}$, Karin Lind ${ }^{1,2}$, and Thomas Nordlander ${ }^{3,4}$ \\ 1 Observational Astrophysics, Division of Astronomy and Space Physics, Department of Physics and Astronomy, Uppsala University, \\ Box 516, 75120 Uppsala, Sweden \\ e-mail: alvin.gavel@physics.uu.se \\ 2 Max Planck Institute for Astronomy (MPIA), Koenigstuhl 17, 69117 Heidelberg, Germany \\ 3 Research School of Astronomy and Astrophysics, Australian National University, Canberra, ACT 2611, Australia \\ 4 ARC Centre of Excellence for All Sky Astrophysics in 3 Dimensions (ASTRO 3D), Australia
}

Received 22 May 2019 / Accepted 10 July 2019

\begin{abstract}
Context. The Gaia-ESO Survey has taken high-quality spectra of a subset of 100000 stars observed with the Gaia spacecraft. The goal for this subset is to derive chemical abundances for these stars that will complement the astrometric data collected by Gaia. Deriving the chemical abundances requires that the stellar parameters be determined.

Aims. We present a pipeline for deriving stellar parameters from spectra observed with the FLAMES-UVES spectrograph in its standard fibre-fed mode centred on $580 \mathrm{~nm}$, as used in the Gaia-ESO Survey. We quantify the performance of the pipeline in terms of systematic offsets and scatter. In doing so, we present a general method for benchmarking stellar parameter determination pipelines. Methods. Assuming a general model of the errors in stellar parameter pipelines, together with a sample of spectra of stars whose stellar parameters are known from fundamental measurements and relations, we use a Markov chain Monte Carlo method to quantitatively test the pipeline.

Results. We find that the pipeline provides parameter estimates with systematic errors on effective temperature below $100 \mathrm{~K}$, on surface gravity below 0.1 dex, and on metallicity below 0.05 dex for the main spectral types of star observed in the Gaia-ESO Survey and tested here. The performance on red giants is somewhat lower.

Conclusions. The pipeline performs well enough to fulfil its intended purpose within the Gaia-ESO Survey. It is also general enough that it can be put to use on spectra from other surveys or other spectrographs similar to FLAMES-UVES.
\end{abstract}

Key words. methods: numerical - stars: atmospheres - stars: statistics

\section{Introduction}

The Gaia spacecraft is currently making high-precision parallax measurements to determine positions and proper motions, together with spectroscopic radial velocity determinations, for astronomical objects in and around the Milky Way. To date, the Gaia sample contains more than 1.7 billion objects (Gaia Collaboration 2018). Between 2012 and 2018, the Gaia-ESO Survey (GES) has performed complementary ground-based observations, taking high-resolution spectra of a subset of about one hundred thousand Gaia stars, together with some stars too bright to be observed by Gaia. These spectra are being used to derive chemical abundances for the GES stars. The intent is for the Gaia astrometric data and the GES chemical data to be used together to study the history of the Milky Way, and by extension galactic evolution in general (Gilmore et al. 2012).

As an initial step in determining chemical abundances for the stars, it is necessary to determine their stellar parameters. For GES, this requires pipelines that automatically perform the determination. This is partly because the sheer number of spectra precludes manual fitting of model spectra, but also because it promotes reproducibility and homogeneity. A spectroscopist who has performed manual fitting of a handful of spectra can hopefully account for the process for each one, but this cannot reasonably be done for thousands of individual spectra. If a data set were to be split between several spectroscopists for manual fitting, the results would also be split into as many clusters with their own systematic errors due to the idiosyncrasies of each individual spectroscopist's fitting process. Since the GES data will be used for statistical analyses, avoiding this clustering becomes more important.

Several different stellar parameter determination pipelines are used within GES. Different pipelines have to be used for spectra observed with different spectrographs, and for different regions of parameter space. In addition, a number of different pipelines are used on the same samples of spectra. This is done partly to increase the accuracy of the derived parameters since it decreases the impact of the assumptions and approximations going into any particular pipeline. Furthermore, the scatter from pipeline to pipeline provides the most robust way of estimating the error on the derived parameters, although it cannot catch systematic errors coming from assumptions and approximations shared by all (Smiljanic et al. 2014).

Spectra observed with the UVES spectrograph (see Sect. 2) are analysed in 13 groups called nodes, each of which has its own pipeline. An outline of the pipelines is given in Smiljanic et al. (2014). The pipelines use the codes ARES (Sousa et al. 2007), BACCHUS (Masseron et al. 2016), DAOSPEC (Stetson \& Pancino 2008), FAMA (Magrini et al. 2013), FERRE, GALA (Mucciarelli et al. 2013), GAUFRE (Valentini et al. 2013), MATISSE (Recio-Blanco et al. 2006), MyGIsFOS (Sbordone et al. 2014), ROTFIT (Frasca et al. 2003, 2006), SME (Piskunov $\&$ Valenti 1996, 2017) and StePar (Tabernero et al. 2013). This paper documents the SME-based pipeline used by the LundUppsala-MPIA-Bordeaux-ANU (LUMBA) node. 


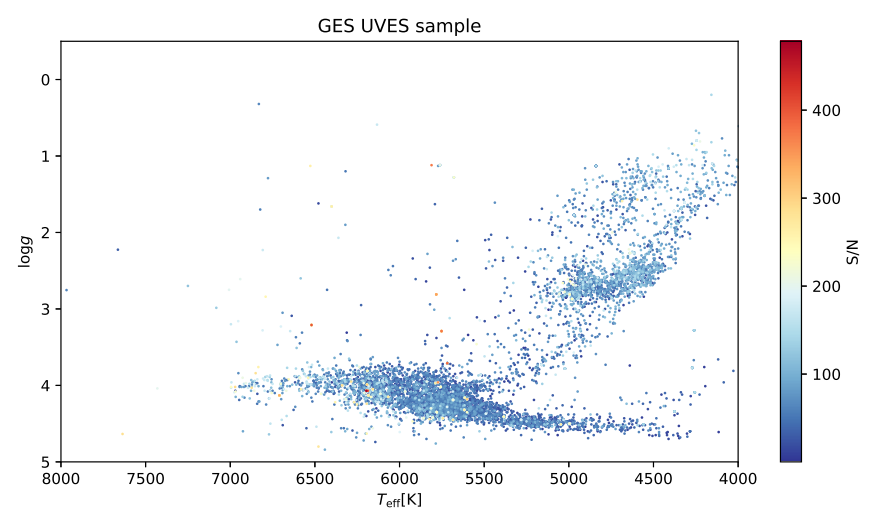

Fig. 1. Spectra analysed with the pipeline described in this paper. The stellar parameters given are the best estimates arrived at by several groups within GES. The spectra are colour-coded according to $\mathrm{S} / \mathrm{N}$. Where stars overlap, those with highest $\mathrm{S} / \mathrm{N}$ have been placed on top.

The portion of the GES sample analysed with this pipeline contains 6941 spectra, excluding the spectra that are used in benchmarking the pipeline. The median $\mathrm{S} / \mathrm{N}$ is 60 . The central $90 \%$ of the $\mathrm{S} / \mathrm{N}$ values for the sample fall in the range $17-180$. We plot the current best GES estimates of $T_{\text {eff }}$ and $\log g$, colourcoded by $\mathrm{S} / \mathrm{N}$ in Fig. 1. These estimates are a homogenisation of estimates produced by several groups within GES, including LUMBA.

The paper is organised as follows. Section 2 gives a brief overview of the characteristics of the observational material collected as part of GES. Section 3 describes the pipeline itself. Section 4 discusses the performance of the pipeline. Section 5 summarises our findings.

\section{Observations with FLAMES-UVES}

The Fibre Large Array Multi Element Spectrograph (FLAMES) is a spectrograph installed on the VLT UT2 telescope (Pasquini et al. 2002). It feeds the red arm of the two-arm cross-dispersed echelle spectrograph called Ultraviolet and Visual Echelle Spectrograph (UVES; Dekker et al. 2000).

The spectra used here have a resolution of around $R \equiv$ $\lambda / \delta \lambda=47000$, and are taken with the RED580 setting, which covers the wavelength range of $4760 \AA-6840 \AA$, with a $50 \AA$ gap in the middle due to the physical gap between the two detectors making up the UVES red arm (Sbordone \& Ledoux 2018).

\section{Pipeline}

The pipeline is based on the LUMBA pipeline for spectra taken with the GIRAFFE spectrograph. They differ in a number of ways, including modifications to make use of the higher resolution and different wavelength range of the UVES. It has been used, in different versions, in the internal GES data releases iDR5 and iDR6. It is similar to the pipeline for the GALAH spectrograph, which is also based on the LUMBA pipeline for GIRAFFE (Buder et al. 2018).

\subsection{Algorithm}

Based on an observed spectrum, the pipeline attempts to fit a synthetic spectrum by varying the following parameters:

1. $T_{\text {eff }}$ : effective temperature;

2. $\log g$ : surface gravity;
3. $[\mathrm{Fe} / \mathrm{H}]$ : metallicity;

4. $v \sin i$ : projected rotational speed of stellar equator;

5. $v_{\text {mic }}:$ microturbulence parameter;

6. $[\mathrm{Ca} / \mathrm{Fe}]$ : calcium abundance;

7. $[\mathrm{Mg} / \mathrm{Fe}]$ : magnesium abundance.

Since the macroturbulence parameter $v_{\text {mac }}$ tends to be almost fully degenerate with $v \sin i$, it is given a separate treatment, described in Appendix A. The fitting is done over discrete segments in the spectrum, which have been selected to contain spectral lines that constrain each parameter. This is described in detail in Sect. 3.2. When running, the pipeline goes through the following four steps:

1. Preliminary normalisation of all segments;

2. Preliminary determination of all parameters, performing at most 2 iterations of the fitting algorithm;

3. Final normalisation of all segments;

4. Final determination of all parameters, performing at most 20 iterations of the fitting algorithm.

This is done using a number of pre-existing software tools, described in Sect. 3.3.

\subsection{Line selection}

Our choice of lines is based on classical spectroscopic techniques to constrain the main stellar parameters $T_{\text {eff }}, \log g,[\mathrm{Fe} / \mathrm{H}]$, and additional parameters like $v \sin i$ and $v_{\text {mic }}$ (see e.g. Gray 2005). However, the goodness-of-fit metric used in the fitting treats all lines equivalently. This means that to the extent that a line has any sensitivity at all to a parameter, it will contribute to the estimate of that parameter. The spectral lines analysed in the pipeline fall into six groups, each of which is intended to predominantly constrain a single stellar parameter:

- Strong neutral calcium lines, which are sensitive to $\log g$ and the calcium abundance;

- Weak neutral calcium lines, which are sensitive to the calcium abundance but not $\log g$;

- Strong neutral magnesium lines, which are sensitive to $\log g$ and the magnesium abundance;

- A set of neutral iron lines, which are sensitive to $[\mathrm{Fe} / \mathrm{H}]$;

- A set of singly ionised iron lines, which are sensitive to $\log g$ and $[\mathrm{Fe} / \mathrm{H}]$.

The weak lines of calcium and magnesium are intended to break the degeneracy in the strong lines between $\log g$ and the abundances. There are no separate lines for $T_{\mathrm{eff}}, v \sin i$, and $v_{\mathrm{mic}}$ since they affect all lines in ways that are not degenerate with the other parameters. While the calcium and magnesium lines are mainly of interest for their usefulness in determining $\log g$, the derived abundances can also be used to identify stars with discrepant chemical compositions (departure of $\alpha$-elements as seen in the thick disk and the halo), even before any detailed abundance analysis has been done.

The lines were selected from the GES line list, based on the quality of their measured transition probabilities and blending properties (Heiter et al. 2015a, 2019). This is described in Sect. 3.3.2. The analysis uses line and segment masks, which is explained in Sect. 3.3.1. We give a full list of the lines in Appendix B.

\subsection{External software}

The central piece of external software in the pipeline is the spectral synthesis code SME. This in turn requires the GES line list, a grid of model atmospheres, and grids of non-local thermodynamic equilibrium (NLTE) departure coefficients. 
The pipeline is written in Interactive Data Language (IDL) and makes extensive use of functions from the IDL Astronomy User's Library (IDLAstro) and the Coyote IDL library (Landsman 1993; Fanning 2015).

\subsubsection{Spectroscopy Made Easy}

Spectroscopy Made Easy (SME) is a code for computing synthetic spectra on the fly, which can be used against observed spectra to automatically fit stellar parameters and/or chemical abundances. It was originally published in 1996. Since then it has been substantially expanded and improved (Piskunov \& Valenti 1996, 2017). At the time of writing the pipeline uses SME version 542, and we plan to update it as new versions are released.

When fitting a spectrum, SME requires the user to define segment masks, continuum masks, and line masks. The segment masks specify which wavelength regions are to be considered at all. Outside, no synthesis and no fitting is performed. The continuum masks specify the regions within each segment that are assumed to be close to the continuum, which is used by SME to normalise the segments. The line masks state the regions within each segment over which the fitting is to be performed (Piskunov et al. 2016). Since they are susceptible to chromospheric contributions and deviations from local thermodynamic equilibrium (LTE), the cores of strong lines are left out of the fit, as described in Appendix C.

SME can normalise an observed spectrum, using any of several procedures. In our case, it is done by rescaling each individual segment under the assumption that the continuum is locally linear. The scaling is done by minimising a Pearson's $\chi^{2}$ sum between the observed and modelled intensities over the pixels covered by the continuum mask (Piskunov et al. 2016). We note that the model spectrum that is computed for the normalisation includes spectral lines. This means that even if spectral lines happen to be covered by the continuum mask, it is possible to get a useful normalisation as long as those lines are included in the line list. The inaccuracy in the normalisation then depends on how close the stellar parameters used to compute the model spectrum are to the correct values. By alternately fitting stellar parameters and normalising, it is then possible to get good estimates of both. If the continuum mask covers lines not included in the line list, on the other hand, it will lead to the continuum being incorrectly fitted. We use the automated algorithm described in Appendix D, which determines a suitable continuum mask for each observed spectrum and segment and takes into account blending and accuracy criteria.

When deriving stellar parameters, SME attempts to minimise a pseudo- $\chi^{2}$ sum, defined as

$\chi^{2}=\sum_{i=0}^{n} \frac{O_{i}\left(O_{i}-E_{i}\right)^{2}}{\sigma_{i}^{2}}$,

where $O_{i}$ is the observed intensity in pixel $i, E_{i}$ is the modelled intensity in the same pixel, $\sigma_{i}$ is the uncertainty in the observed intensity, and the sum runs over all pixels contained within the line masks. The minimisation is done using a version of the Levenberg-Marquardt algorithm, adapted to the modified $\chi^{2}$ sum (Levenberg 1944; Marquardt 1963; Piskunov \& Valenti 2017). The additional factor of $O_{i}$, which is not present in a conventional $\chi^{2}$ sum, causes SME to prefer placing the model spectrum above the observed spectrum than to placing it below. This reflects a heuristic commonly used by spectroscopists when fitting spectra manually; the main source of error in a synthetic spectrum often comes from the incompleteness of the line list.
This means that a perfect choice of stellar parameters should produce a model spectrum lying slightly above the observed spectrum (Piskunov, priv. comm.).

Accurately determining errors in fitted stellar parameters is still an unsolved problem. Most error estimations assume that the main source of error is statistical errors on the input data. This is almost never the case for spectral fitting, where the error is typically dominated by systematic errors on the model. SME versions from 433 onwards use a heuristic method for estimating the error that tries to take systematics into account. All pixels $i$ in the line mask with a non-zero partial derivative of the model flux $F_{i}$ with respect to the parameter $p$ are chosen. Of the chosen pixels, those with a residual $R_{i}$ between the observed and the best-fit model spectrum that is less than five times the measurement uncertainty are then selected. Under the assumption that the model intensity is locally linear, the change $\Delta p$ needed to make the residual zero is given by

$\Delta p=\frac{R_{i}}{\partial F_{i} / \partial p}$.

This gives a distribution of $\Delta p$-values. The width of this distribution is taken to be the $68 \%$ of values around the centre. This width is then taken to be the best estimate of the parameter uncertainty (Piskunov \& Valenti 2017). This method seems to overestimate the errors by a factor of a few, but it is still useful as an order-of-magnitude estimate.

The fitting procedure requires a set of starting values for the stellar parameters. As long as the masks are chosen so that the problem is not close to being degenerate, these starting values will generally only affect the time needed for the fitting to converge on a solution, and will not affect the final result (Piskunov $\&$ Valenti 2017). The only exception is radial velocity, for which it is necessary to supply a good starting estimate (Piskunov, priv. comm.). For GES spectra this is usually irrelevant, since most of them have been corrected for radial velocity. The starting values used for the other parameters in the pipeline are generally the best estimates given in the internal data releases of GES. The exception is the microturbulence parameter $v_{\text {mic }}$, which together with the fixed macroturbulence parameter $v_{\text {mac }}$ is given the treatment described in Appendix E. The abundance pattern is initially assumed to be the same as in the Sun, although rescaled based on the starting value of $[\mathrm{Fe} / \mathrm{H}]$ (Piskunov et al. 2016). In the pipeline, the individual abundances for calcium and magnesium are free parameters since lines of those elements are used to estimate $\log g$.

\subsubsection{GES line list}

To compute spectra, SME needs a list specifying the wavelength, oscillator strength, and other line parameters for the spectral lines thought to be relevant. For this pipeline we used the GES line list, which was developed to enable homogeneous analysis with high-quality line data within GES. It contains the best literature values of the line parameters for 1341 transitions.

Before the line and segment masks were defined, the reliability of the atomic data and their degree of blending with other lines were both classified into three categories of decreasing quality: Y, U, and N. The atomic data was classified according to the assumed reliability of the literature values. Recent precise laboratory measurements were classified as Y. Older less precise laboratory measurements were classified as U. Semi-empirical calculations were classified as $\mathrm{N}$. The blending was classified by comparing model spectra to reference spectra for the Sun and Arcturus. Lines only blended with lines of the same species in 
both stars were classified as Y. Lines blended to some extent with other species in at least one of the stars were classified as U. Lines strongly blended with other species in both stars were classified as N (Heiter et al. 2015a, 2019). The line and segment masks were then defined, when possible, by selecting lines of quality $\mathrm{Y}$ in both respects. When a line type only had a few available lines, lines of quality $U$ in either reliability or blending, but not both, were permitted.

\subsubsection{MARCS model atmospheres}

To compute spectra, SME needs a grid of model atmospheres over the relevant ranges of $T_{\text {eff }}, \log g$, and $[\mathrm{Fe} / \mathrm{H}]$. For our pipeline we use a grid calculated by Bengt Edvardsson using the Model Atmospheres with a Radiative and Convective Scheme (MARCS) code, within the ranges $4000 \mathrm{~K}<T_{\text {eff }}<8000 \mathrm{~K}$, $-0.5<\log g<5.0,-5.0<[\mathrm{Fe} / \mathrm{H}]<1.0$.

MARCS has been in use in different versions since the 1970s, and a standardised version was published in 2008 . It computes model atmospheres for late-type stars under the assumptions of plane-parallel or spherical symmetry, hydrostatic equilibrium, mixing-length convection treatment, and LTE (Gustafsson et al. 2008).

\subsubsection{NLTE grids}

While the MARCS model atmospheres were computed under the assumption of LTE radiative transfer, SME performs spectrum synthesis taking into account pre-computed grids of NLTE departure coefficients for certain elements (Piskunov \& Valenti 2017). At present, the pipeline uses departure coefficients only for iron (Bergemann et al. 2012; Lind et al. 2012).

\section{Performance}

We now attempt to evaluate how well the pipeline performs in practice. Since the pipeline is mainly used within GES for statistical analysis of large samples of stars, we are mostly interested in the systematic offsets in the derived parameters. These offsets are expected to differ somewhat depending on the type of star. For our purposes, we consider that the pipeline performs well for a type of star as long as the offset in $T_{\text {eff }}$ is below $100 \mathrm{~K}$, the offset in $\log g$ is below $0.1 \mathrm{dex}$, and the offset in $[\mathrm{Fe} / \mathrm{H}]$ is below 0.05 dex. These offsets are somewhat arbitrarily chosen, but reflect requirements from science cases related to Galactic chemical evolution.

The most straightforward way of estimating the offsets is by using the pipeline to derive stellar parameters of stars for which the correct values are already known. We have a sample of such stars called the "Gaia FGK benchmark stars", widely used within GES for this purpose (Blanco-Cuaresma et al. 2014; Heiter et al. 2015b). As an additional sanity check, we test the pipeline on the open cluster M 67, comparing the derived stellar parameters to stellar isochrones.

\subsection{Comparison to benchmark stars}

For the Gaia FGK benchmark stars, $T_{\text {eff }}$ and $\log g$ have been estimated from fundamental observations and relations, such as direct measurements of angular diameters, astrometric parallax, and the bolometric flux. This makes the estimates independent of the assumptions made in spectral modelling (Blanco-Cuaresma et al. 2014; Heiter et al. 2015b). The benchmark stars used in
Table 1. Benchmark stars.

\begin{tabular}{lll}
\hline \hline Star type & Star & Spectra \\
\hline \multirow{5}{*}{ Solar-type stars } & The Sun & 9 \\
& 18 Sco & 3 \\
& $\mu$ Ara & 3 \\
& $\mu$ Cas A & 1 \\
& $\alpha$ Cen A & 2 \\
& $\tau$ Cet & 3 \\
& $\beta$ Vir & 3 \\
& HD 22879 & 2 \\
\hline \multirow{5}{*}{ F dwarfs } & Procyon & 6 \\
& HD 49933 & 2 \\
& HD 84937 & 5 \\
\hline \multirow{5}{*}{ FGK subgiants } & $\eta$ Boo & 2 \\
& $\delta$ Eri & 4 \\
& $\epsilon$ For & 1 \\
& $\beta$ Hyi & 3 \\
& HD 140283 & 5 \\
\hline \multirow{5}{*}{ Red giants } & Arcturus & 5 \\
& $\beta$ Ara & 1 \\
& $\xi$ Hya & 2 \\
& $\mu$ Leo & 2 \\
& $\epsilon$ Vir & 3 \\
& HD 107328 & 2 \\
& HD 220009 & 2 \\
\hline & & \\
& &
\end{tabular}

this paper are were chosen to be representative of four types of stars, referred to as

- Solar-type stars;

- F dwarfs;

- FGK subgiants;

- Red giants.

For the first three types, we used all of the GES benchmark stars available. For the red giants, we removed all stars with $T_{\text {eff }}<4000 \mathrm{~K}$ since they fall outside of our grid of atmospheres. We also disregarded the star HD 122563, which our pipeline is unable to analyse. We believe that this occurs because the star both is a red giant and has a metallicity of $[\mathrm{Fe} / \mathrm{H}]=-2.64$, the lowest of all benchmark stars. Stars with very low metallicity are in general difficult to analyse spectroscopically since the low value decreases the depth of spectral lines. Red giants are also difficult to analyse with this particular pipeline, for reasons we discuss below. In Table 1, we list the benchmark stars used together with the number of spectra available for each star.

The benchmark spectra have been observed using the ESPaDOnS, NARVAL, and UVES spectrographs and convolved to a resolution of 47000 , matching that of FLAMESUVES (Blanco-Cuaresma et al. 2014). For these spectrographs the gaps in the spectra do not overlap with the segments used in our analysis. However, the wavelength gap around 5306-5337 A in the HARPS benchmark spectra contains several FeIII lines used in our analysis, which has forced us to avoid those spectra. The spectral files come from the Blanco-Cuaresma library in Blanco-Cuaresma (2019).

Using the method described in Appendix F, we estimated the systematic offsets for each parameter and type of star. We show our best estimates in Table 2 . We note that unfortunately it is not possible to directly compare the performance to that of similar pipelines. The method for estimating performance was developed for this paper, and has not yet been used on other pipelines. 
Table 2. Estimated systematic offsets for benchmark stars.

\begin{tabular}{llll}
\hline \hline Star type & $\Delta T_{\text {eff }} / \mathrm{K}$ & $\log g$ & {$[\mathrm{Fe} / \mathrm{H}]$} \\
\hline The Sun & $-25 \pm 4$ & $-0.070 \pm 0.004$ & $0.008 \pm 0.003$ \\
Solar-type & $-30 \pm 40$ & $-0.04 \pm 0.03$ & $0.003 \pm 0.004$ \\
F dwarfs & $50 \pm 40$ & $0.08 \pm 0.05$ & $0.019 \pm 0.008$ \\
FGK subg. & $0 \pm 20$ & $0.02 \pm 0.03$ & $-0.03 \pm 0.02$ \\
Red giants & $50 \pm 20$ & $0.15 \pm 0.01$ & $-0.07 \pm 0.05$ \\
\hline
\end{tabular}

Notes. We show the best estimates of the systematic offsets (derived benchmark) and their $1 \sigma$ uncertainty values in each parameter for each type of benchmark star. We also show this for the Sun alone.

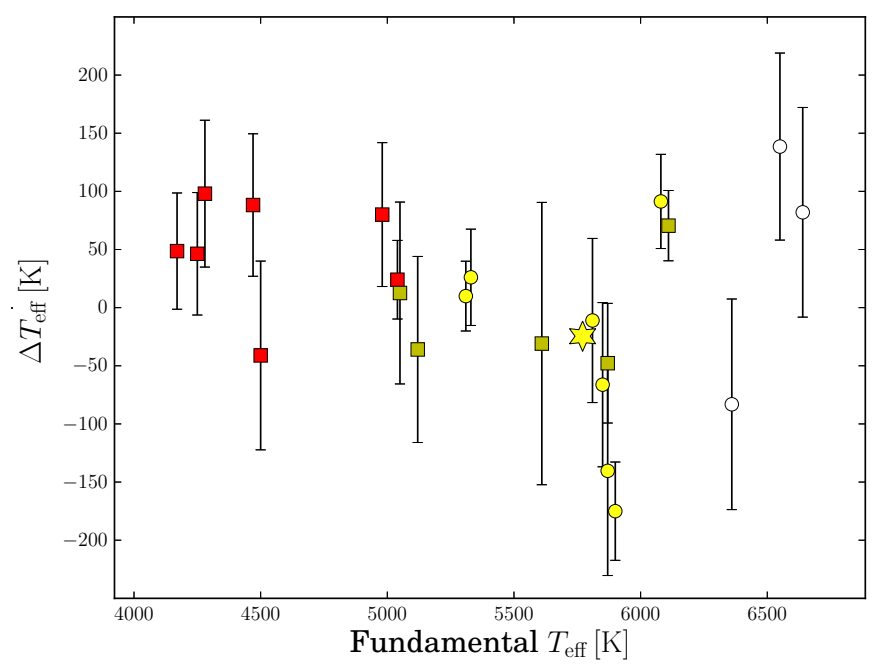

Fig. 2. Unweighted mean offset for $T_{\text {eff }}$ for benchmark stars. Error bars denote the standard deviation in the mean added in quadrature to the uncertainty in the fundamental-parameter estimate provided in Heiter et al. (2015b). The yellow hexagram denotes the Sun. Yellow circles denote other solar-type stars. White circles denote F dwarfs. Yellow squares denote FGK subgiants. Red squares denote red giants.

We show the mean offset for each individual star, averaged over all spectra of that star, for $T_{\text {eff }}$ in Fig. 2, for $\log g$ in Fig. 3, and for $[\mathrm{Fe} / \mathrm{H}]$ in Fig. 4.

As can be seen, the pipeline generally performs satisfactorily, with systematic offsets in $T_{\text {eff }}$ below $100 \mathrm{~K}$, in $\log g$ below $0.1 \mathrm{dex}$, and in $[\mathrm{Fe} / \mathrm{H}]$ below $0.05 \mathrm{dex}$. The only exception is the group of red giants for which the average offsets in $\log g$ and $[\mathrm{Fe} / \mathrm{H}]$ are estimated to be $0.15 \pm 0.03 \mathrm{dex}$ and $-0.07 \pm 0.05 \mathrm{dex}$, respectively. We believe that this is partly due to the increased line density at lower temperatures causing line blending, which makes the continuum determination difficult. However, since red giants differ the most from the stars used for testing during the early development of the pipeline, it may be that they conform less to other implicit assumptions built into the pipeline. We note that the tendency is to overestimate $\log g$ for red giants, which is the opposite of what usually results from LTE-based stellar parameter determinations.

\subsection{Comparison to M 67 isochrones}

While benchmark stars give the most reliable and easily interpreted tests of a stellar parameter pipeline, it is possible to also use stellar clusters for additional sanity checks. We chose the old open cluster M 67 (NGC 2682) for this. This cluster is well studied in the literature and contains stars with a wide span of stellar

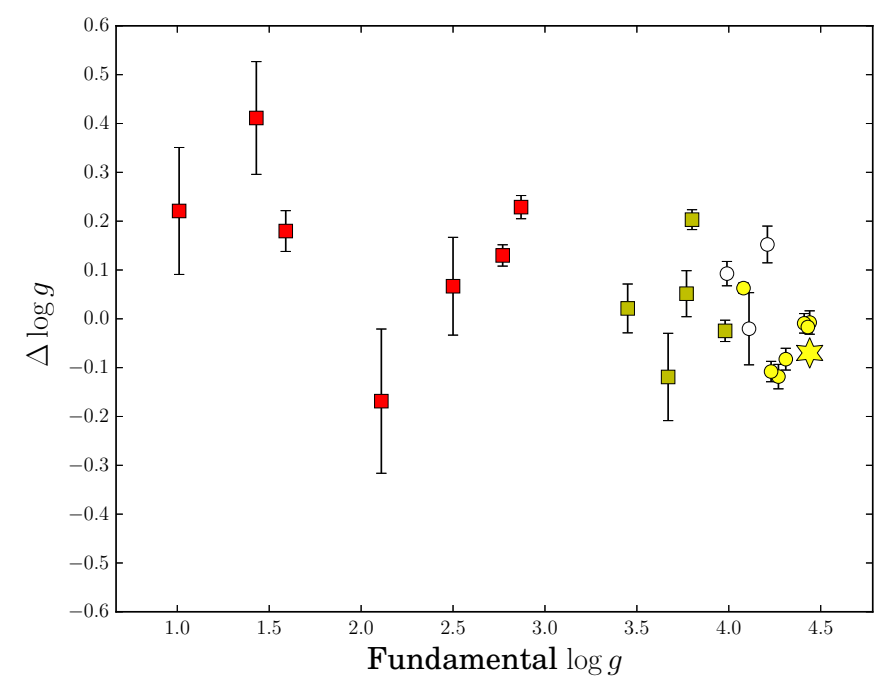

Fig. 3. Unweighted mean offset for $\log g$ for benchmark stars. Error bars denote the standard deviation in the mean added in quadrature to the uncertainty in the fundamental-parameter estimate provided in Heiter et al. (2015b), the other symbols as in Fig. 2.

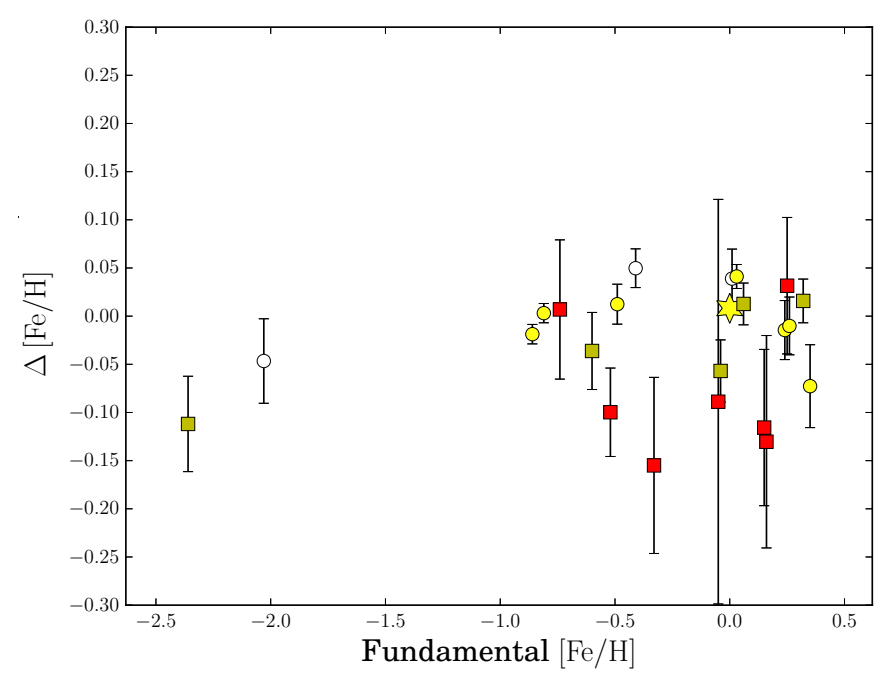

Fig. 4. Unweighted mean offset for $[\mathrm{Fe} / \mathrm{H}]$ for benchmark stars. Error bars denote the standard deviation in the mean added in quadrature to the uncertainty in the fundamental-parameter estimate provided in Heiter et al. (2015b), the other symbols as in Fig. 2.

parameters, including the solar twin YBP 1194 (Önehag et al. 2011) and some blue stragglers.

We have access to 42 UVES spectra of stars in the field of the cluster, all of which come from the GES sample. Of these, 20 were observed in 2004 within project 072.D-0309(A) with principal investigator C.H.F. Melo, and 22 were observed in 2009 within project 082.D-0726(A) with principal investigator B. Gustafsson. All of the spectra are in the ESO archive, and reprocessed spectra will be made available after the final GES data release. The stellar parameters derived by LUMBA, and the other nodes mentioned in Sect. 1, will be included in the final GES data release.

Based on the description in the SIMBAD database, we listed some of the stars as "red giant", "variable", "binary", and "blue straggler" (Wenger et al. 2000). The spectra also include the solar twin YBP-1194. This could in principle be a very useful reference star since it has been repeatedly studied over the 


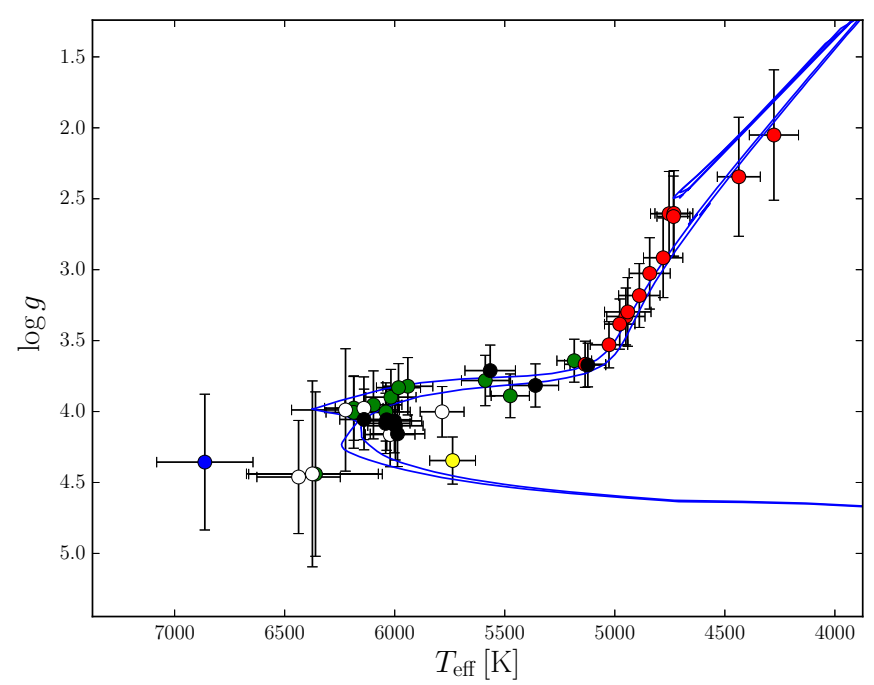

Fig. 5. Estimates of $T_{\text {eff }}$ and $\log g$ for stars in $\mathrm{M}$ 67. Error bars denote the errors returned by SME. The yellow circle denotes the solar twin YBP1194. Blue circles denote blue stragglers. Green circles denote variable stars. White circles denote binary stars. Red circles denote red giants. Black circles denote other stars. The blue curves denote isochrones calculated for the highest and lowest age estimates for the cluster. One star with $\sigma_{T_{\text {eff }}}>500 \mathrm{~K}$ has not been included.

past few years (Liu et al. 2016). Unfortunately, there are known problems with the spectra of this star taken with FLAMESUVES (Önehag et al. 2011). We list the coordinates for these stars in Table G.1, together with our classification.

We compare the derived $T_{\text {eff }}$ and $\log g$ to isochrones with appropriately chosen metallicity and age, and look for trends in $[\mathrm{Fe} / \mathrm{H}]$ as a function of $\log g$. The cluster isochrones were calculated using PARSEC v1.2S and COLIBRI S_35 using version 3.2 of the web input form ${ }^{1}$ (Marigo et al. 2017). We calculated isochrones for ages $3.5 \mathrm{~Gy}$ and $4.8 \mathrm{~Gy}$ since the true age is believed to lie somewhere within this range. We took the metallicity to be 0.06 dex (Önehag et al. 2014).

We show the $T_{\text {eff }}$ and $\log g$ estimates and isochrones in Fig. 5. Visually, the agreement looks good. The only stars that are noticeably far off from the isochrone are the blue straggler, two binary star systems, and one variable star. However, it is difficult to make any quantitative statement of how good the agreement is. We note that the estimates on average fall within $0.6 \sigma$ of the closest points on the two isochrones, but this metric cannot be easily turned into an estimate of the parameter offsets. To calculate the likelihood of getting a particular set of estimates, assuming certain values of the parameter offsets requires us to have a well-defined prior for both the cluster age and the positions on the isochrone. For a detailed discussion of the problems with comparing isochrones to data, see Valls-Gabaud (2014).

The estimates of $[\mathrm{Fe} / \mathrm{H}]$ have a weighted mean of $-0.02 \pm$ 0.02 dex, which is somewhat lower than the assumed cluster $[\mathrm{Fe} / \mathrm{H}]$. This mean is independent of whether red giants are included or not. However, the cluster $[\mathrm{Fe} / \mathrm{H}]$ was adopted from Önehag et al. (2014) after correcting for the effects of atomic diffusion. Considering this, our $[\mathrm{Fe} / \mathrm{H}]$ estimate for the mainsequence, turn-off, and subgiant stars agrees with Önehag et al. (2014).

We plot the estimated $[\mathrm{Fe} / \mathrm{H}]$ as a function of the estimated $\log g$ together with a Deming regression in Fig. 6. There is an apparent trend in $[\mathrm{Fe} / \mathrm{H}]$, but it is only significant if red giants are

\footnotetext{
1 http://stev.oapd.inaf.it/cgi-bin/cmd
}

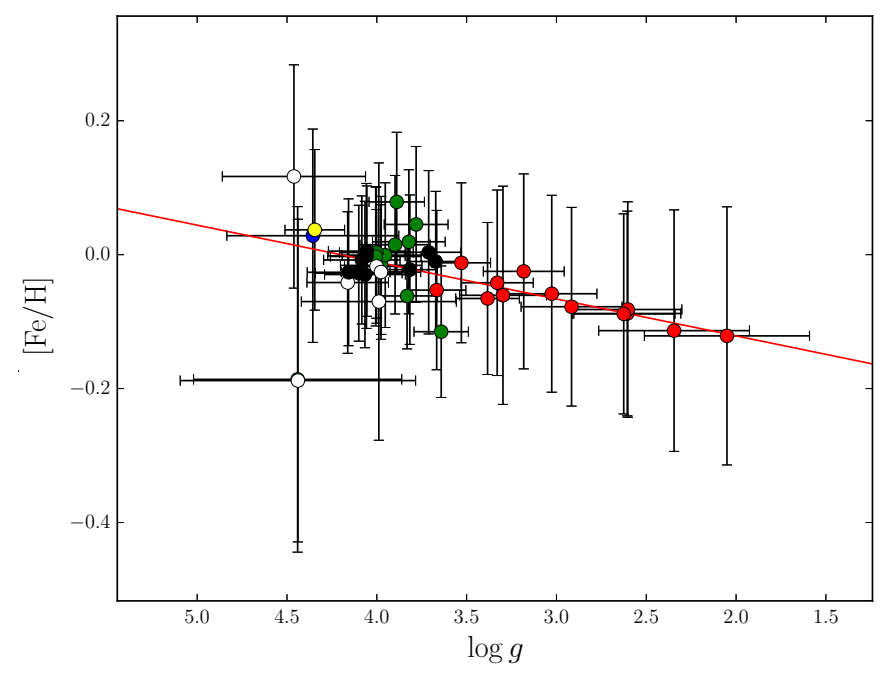

Fig. 6. Estimates of $\log g$ and $[\mathrm{Fe} / \mathrm{H}]$ for stars in M 67. Error bars denote the errors returned by SME. The yellow circle denotes YBP-1194. Blue circles denote blue stragglers. Green circles denote variable stars. White circles denote binary stars. Red circles denote red giants. Black circles denote other stars. The red line denotes the best-fit line given by the Deming regression. One star with $\sigma_{T_{\text {eff }}}>500 \mathrm{~K}$ has not been included.

included in the fit. The trend is not what is expected from atomic diffusion and thus contradicts recent findings (Souto et al. 2019). Given the above discussions on the performance of the pipeline on red-giant spectra, we do not believe that this trend reflects the true iron trend in M 67. Similar difficulties with analysing red giants were seen when M 67 was studied with the related GALAH pipeline (Gao et al. 2018).

\section{Summary}

As part of the work within GES, the LUMBA collaboration has developed a pipeline for deriving stellar parameters from spectra observed with FLAMES-UVES or similar spectrographs. The pipeline uses the spectral synthesis code SME to do the fitting, together with the GES line list, a grid of MARCS model atmospheres, NLTE grids, and commonly used IDL libraries. The best-fit parameters are determined by minimising a $\chi^{2}$-like sum.

We developed a method for estimating the systematic offsets for stellar parameter pipelines by testing them on the spectra of benchmark stars with stellar parameters estimated from fundamental observations and relations. Using this method reveals systematic offsets in $T_{\text {eff }}$ below $100 \mathrm{~K}$, in $\log g$ below 0.1 dex, and in $[\mathrm{Fe} / \mathrm{H}]$ below 0.05 dex for the most common GES targets. The performance is somewhat degraded for red giants. We comment on the difficulties of using stellar clusters as quantitative tests of pipeline performance. The achieved level of accuracy makes the pipeline suitable for applications in the framework of studies of Galactic chemical evolution.

Acknowledgements. We wish to commemorate our colleague and friend Gregory Ruchti (1980-2019) who originally branched off this pipeline from the LUMBA-internal GIRAFFE pipeline. We thank the GES team for the work behind the M 67 spectra and for making them available to us. We thank Caroline Soubiran, Paula Jofré, and Maria Bergemann for contributions to the work behind the pipeline. AG, UH, and AJK acknowledge support from the Swedish National Space Agency and the Knut and Alice Wallenberg Foundation. Parts of this research were conducted by the Australian Research Council Centre of Excellence for All Sky Astrophysics in 3 Dimensions (ASTRO 3D), through project number CE170100013. PG thanks the European Science Foundation (ESF) for support in the framework of EuroGENESIS. 


\section{A. Gavel et al.: LUMBA UVES pipeline}

KL acknowledges funds from the Alexander von Humboldt Foundation in the framework of the Sofja Kovalevskaja Award endowed by the Federal Ministry of Education and Research and funds from the Swedish Research Council (Grant no. 2015-00415_3) and Marie Skłodowska Curie Actions (Cofund Project INCA 600398). This research has made use of the SIMBAD database, operated at CDS, Strasbourg, France.

\section{References}

Bergemann, M., Lind, K., Collet, R., Magic, Z., \& Asplund, M. 2012, MNRAS, 427, 27

Blanco-Cuaresma, S. 2019, The Gaia FGK Benchmark Stars, https://www . blancocuaresma.com/s/benchmarkstars

Blanco-Cuaresma, S., Soubiran, C., Jofré, P., \& Heiter, U. 2014, A\&A, 566, A98

Buder, S., Asplund, M., Duong, L., et al. 2018, MNRAS, 478, 4513

Dekker, H., D'Odoric, S., Kaufer, A., Delabre, B., \& Kotzlowski, H. 2000, in Optical and IR Telescope Instrumentation and Detectors, Proc. SPIE, 4008

Fanning, D. W. 2015, Coyote's Guide to IDL Programming, http://www. idlcoyote.com/

Foreman-Mackey, D., Hogg, D. W., Lang, D., \& Goodman, J. 2013, PASP, 125, 306

Frasca, A., Alcalá, J. M., Covino, E., et al. 2003, A\&A, 405, 149

Frasca, A., Guillout, P., Marilli, E., et al. 2006, A\&A, 454, 301

Gaia Collaboration (Brown, A. G. A., et al.) 2018, A\&A, 616, A1

Gao, X., Lind, K., Amarsi, A. M., et al. 2018, MNRAS, 481, 2666

Gilmore, G., Randich, S., Asplund, M., et al. 2012, The Messenger, 147, 25

Gray, D. F. 2005, The Observation and Analysis of Stellar Photospheres, 3rd edn (Cambridge: Cambridge University Press)

Gustafsson, B., Edvardsson, B., Eriksson, K., et al. 2008, A\&A, 486, 951

Heiter, U., Lind, K., Asplund, M., et al. 2015a, Phys. Scr., 90, 054010

Heiter, U., Jofré, P., Gustafsson, B., et al. 2015b, A\&A, 582, A49

Heiter, U., Lind, K., Bergemann, M., et al. 2019, A\&A, submitted

Landsman, W. B. 1993, in Astronomical Data Analysis Software and Systems II, eds. R. J. Hanisch, R. J. V. Brissenden, \& J. Barnes, ASP Conf. Ser., 52, 246
Levenberg, K. 1944, Q. Appl. Math., 2, 164

Lind, K., Bergemann, M., \& Asplund, M. 2012, MNRAS, 427, 50

Liu, F., Asplund, M., Yong, D., et al. 2016, MNRAS, 463, 696

Magrini, L., Randich, S., Friel, E., et al. 2013, A\&A, 558, A38

Marigo, P., Girardi, L., Bressan, A., et al. 2017, ApJ, 835, 77

Marquardt, D. W. 1963, J. Soc. Ind. Appl. Math., 11, 431

Masseron, T., Merle, T., \& Hawkins, K. 2016, Astrophysics Source Code Library [record ascl:1605.004]

Mucciarelli, A., Pancino, E., Lovisi, L., Ferraro, F. R., \& Lapenna, E. 2013, ApJ, 766, 78

Önehag, A., Korn, A., Gustafsson, B., Stempels, E., \& VandenBerg, D. A. 2011, A\&A, 528, A85

Önehag, A., Gustafsson, B., \& Korn, A. 2014, A\&A, 562, A102

Pasquini, L., Avila, G., Blecha, A., et al. 2002, The Messenger, 110, 1

Piskunov, N. E., \& Valenti, J. A. 1996, A\&AS, 118, 595

Piskunov, N. E., \& Valenti, J. A. 2017, A\&A, 597, A16

Piskunov, N. E., Valenti, J. A., \& Heiter, U. 2016, Spectroscopy Made "Easy" (SME) User Handbook

Recio-Blanco, A., Bijaoui, A., \& de Laverny, P. 2006, MNRAS, 370, 141

Sbordone, L., \& Ledoux, C. 2018, Very Large Telescope Paranal Science Operations UV-Visual Echelle Spectrograph User manual, 102nd edn., KarlSchwarzschild Str. 2, D-85748 Garching bei München

Sbordone, L., Caffau, E., Bonifacio, P., \& Duffau, S. 2014, A\&A, 564, A109

Smiljanic, R., Korn, A. J., Bergemann, M., et al. 2014, A\&A, 570, A122

Sousa, S. G., Santos, N. C., Israelian, G., Mayor, M., \& Monteiro, M. J. P. F. G. 2007, A\&A, 469, 783

Souto, D., Allende Prieto, C., Cunha, K., et al. 2019, ApJ, 874, 97

Stetson, P. B., \& Pancino, E. 2008, PASP, 120, 1332

Tabernero, H. M., González Hernández, J. I., \& Montes, D. 2013, in Highlights of Spanish Astrophysics VII, eds. J. C. Guirado, L. M. Lara, V. Quilis, \& J. Gorgas, 673

Valenti, J. A., \& Fischer, D. A. 2005, ApJS, 159, 141

Valentini, M., Morel, T., Miglio, A., Fossati, L., \& Munari, U. 2013, Eur. Phys. J. Web Conf., 43, 03006

Valls-Gabaud, D. 2014, EAS Publ. Ser., 65, 225

Wenger, M., Ochsenbein, F., Egret, D., et al. 2000, A\&AS, 143, 9 


\section{Appendix A: Treatment of $v_{\text {mac }}$}

For most spectra, $v \sin i$ and $v_{\mathrm{mac}}$ are too degenerate to be determined separately. Hence, it is common to have a single parameter that essentially stands in for the combined effect of the two parameters. This is done in e.g. Valenti \& Fischer (2005).

In our pipeline we define such a parameter, which we refer to here as $v \sin i_{\text {pseudo. }}$. The starting value of $v \sin i_{\text {pseudo, start }}$ is taken by adding the estimate of $v_{\text {mac }}$ to the starting guess of the pure $v \sin i$ in quadrature. Meanwhile $v_{\text {mac }}$ is treated as being zero in the code:

$v \sin i_{\text {pseudo, start }} \equiv \sqrt{\left(v \sin i_{\text {start }}\right)^{2}+\left(v_{\text {mac }}\right)^{2}}$.
After the fitting is finished, the best estimate $v \sin i_{\text {final }}$ is taken by subtracting in quadrature the estimated $v_{\text {mac }}$ (see Appendix E) from the final estimate of $v \sin i_{\text {pseudo. }}$.

$$
v \sin i_{\text {final }}=\sqrt{\left(v \sin i_{\text {pseudo, final }}\right)^{2}-\left(v_{\text {mac }}\right)^{2}}
$$

\section{Appendix B: Lines}

We show the full list of lines in Table B.1. The "line mask" columns give the wavelength ranges used in the fitting. The "segment" columns give the wavelength ranges of the segments that make up the synthetic spectra.

Table B.1. Line masks.

\begin{tabular}{|c|c|c|c|c|c|c|c|}
\hline \multirow[t]{2}{*}{ Line centre } & \multicolumn{2}{|l|}{ Line mask } & \multicolumn{2}{|l|}{ Segment } & \multirow{2}{*}{$\begin{array}{l}\log g f \\
\text { quality flag }\end{array}$} & \multirow{2}{*}{$\begin{array}{l}\text { Blend } \\
\text { quality flag }\end{array}$} & \multirow[t]{2}{*}{ Species } \\
\hline & Start & End & Start & End & & & \\
\hline $5217.3893 \AA$ & $5216.80 \AA$ & $5217.60 \AA$ & $5241.01 \AA$ & $5251.50 \AA$ & $\mathrm{Y}$ & $\mathrm{Y}$ & $\mathrm{Fe} \mathrm{I}$ \\
\hline $5242.4907 \AA$ & $5242.30 \AA$ & $5242.80 \AA$ & $5261.01 \AA$ & $5274.00 \AA$ & Y & Y & $\mathrm{Fe} I$ \\
\hline $5379.5736 \AA$ & $5379.30 \AA$ & $5379.85 \AA$ & $5390.01 \AA$ & $5399.00 \AA$ & Y & $\mathrm{Y}$ & $\mathrm{Fe} \mathrm{I}$ \\
\hline $5398.2791 \AA$ & $5398.00 \AA$ & $5398.55 \AA$ & $5412.01 \AA$ & $5418.00 \AA$ & $\mathrm{U}$ & $\mathrm{Y}$ & $\mathrm{Fe} I$ \\
\hline $5434.5235 \AA$ & $5434.00 \AA$ & $5434.82 \AA$ & $5440.01 \AA$ & $5449.00 \AA$ & Y & $\mathrm{Y}$ & $\mathrm{Fe} \mathrm{I}$ \\
\hline $5445.0420 \AA$ & $5444.80 \AA$ & $5445.40 \AA$ & $5450.01 \AA$ & $5460.00 \AA$ & U & Y & $\mathrm{Fe} \mathrm{I}$ \\
\hline $5455.4540 \AA$ & $5455.00 \AA$ & $5456.00 \AA$ & $5500.01 \AA$ & $5508.00 \AA$ & $\mathrm{Y}$ & $\mathrm{Y}$ & $\mathrm{Fe} \mathrm{I}$ \\
\hline $5501.4649 \AA$ & $5501.10 \AA$ & $5501.80 \AA$ & $5526.01 \AA$ & $5530.00 \AA$ & $\mathrm{Y}$ & $\mathrm{Y}$ & $\mathrm{Fe} \mathrm{I}$ \\
\hline $5543.9356 \AA$ & $5543.50 \AA$ & $5544.40 \AA$ & $5565.01 \AA$ & $5573.00 \AA$ & $\mathrm{U}$ & $\mathrm{Y}$ & $\mathrm{Fe} \mathrm{I}$ \\
\hline $5569.6180 \AA$ & $5569.10 \AA$ & $5570.10 \AA$ & $5573.01 \AA$ & $5578.00 \AA$ & $\mathrm{Y}$ & $\mathrm{Y}$ & $\mathrm{Fe} \mathrm{I}$ \\
\hline $5576.0888 \AA$ & $5575.60 \AA$ & $5576.60 \AA$ & $5578.01 \AA$ & $5584.00 \AA$ & $\mathrm{Y}$ & $\mathrm{Y}$ & $\mathrm{Fe} \mathrm{I}$ \\
\hline $5586.7555 \AA$ & $5586.46 \AA$ & $5587.30 \AA$ & $5584.01 \AA$ & $5591.00 \AA$ & $\mathrm{Y}$ & $\mathrm{Y}$ & $\mathrm{Fe} \mathrm{I}$ \\
\hline $5679.0229 \AA$ & $5678.77 \AA$ & $5679.41 \AA$ & $5699.01 \AA$ & $5707.00 \AA$ & $\mathrm{Y}$ & $\mathrm{Y}$ & $\mathrm{Fe} \mathrm{I}$ \\
\hline $5701.5442 \AA$ & $5701.35 \AA$ & $5701.75 \AA$ & $5699.01 \AA$ & $5707.00 \AA$ & $\mathrm{Y}$ & Y & $\mathrm{Fe} \mathrm{I}$ \\
\hline $5705.4642 \AA$ & $5705.20 \AA$ & $5705.70 \AA$ & $5853.01 \AA$ & $5861.00 \AA$ & $\mathrm{Y}$ & $\mathrm{Y}$ & $\mathrm{Fe} \mathrm{I}$ \\
\hline $5775.0805 \AA$ & $5774.90 \AA$ & $5775.30 \AA$ & $5865.01 \AA$ & $5870.00 \AA$ & $\mathrm{Y}$ & $\mathrm{Y}$ & $\mathrm{Fe} I$ \\
\hline $5855.0758 \AA$ & $5854.85 \AA$ & $5855.25 \AA$ & $5915.01 \AA$ & $5917.00 \AA$ & $\mathrm{Y}$ & $\mathrm{Y}$ & $\mathrm{Fe} \mathrm{I}$ \\
\hline $5916.2473 \AA$ & $5916.10 \AA$ & $5916.40 \AA$ & $6000.01 \AA$ & $6007.00 \AA$ & $\mathrm{Y}$ & $\mathrm{Y}$ & $\mathrm{Fe} \mathrm{I}$ \\
\hline $5956.6940 \AA$ & $5956.50 \AA$ & $5956.90 \AA$ & $6021.01 \AA$ & $6030.00 \AA$ & $\mathrm{Y}$ & Y & $\mathrm{Fe} \mathrm{I}$ \\
\hline $6003.0111 \AA$ & $6002.80 \AA$ & $6003.20 \AA$ & $6146.00 \AA$ & $6153.00 \AA$ & $\mathrm{Y}$ & Y & $\mathrm{Fe} \mathrm{I}$ \\
\hline $6027.0508 \AA$ & $6026.91 \AA$ & $6027.18 \AA$ & $6146.00 \AA$ & $6153.00 \AA$ & $\mathrm{Y}$ & Y & $\mathrm{Fe} \mathrm{I}$ \\
\hline $6151.6173 \AA$ & $6151.40 \AA$ & $6151.80 \AA$ & $6160.01 \AA$ & $6171.00 \AA$ & $\mathrm{Y}$ & $\mathrm{Y}$ & $\mathrm{Fe} \mathrm{I}$ \\
\hline $6165.3598 \AA$ & $6165.20 \AA$ & $6165.50 \AA$ & $6160.01 \AA$ & $6171.00 \AA$ & $\mathrm{Y}$ & $\mathrm{Y}$ & $\mathrm{Fe} \mathrm{I}$ \\
\hline 6173.3343 A & $6173.05 \AA$ & $6173.65 \AA$ & $6239.01 \AA$ & $6241.50 \AA$ & $\mathrm{Y}$ & Y & $\mathrm{Fe} \mathrm{I}$ \\
\hline $6200.3125 \AA$ & $6200.01 \AA$ & $6200.61 \AA$ & $6242.01 \AA$ & $6258.00 \AA$ & $\mathrm{Y}$ & Y & $\mathrm{Fe} \mathrm{I}$ \\
\hline $6219.2805 \AA$ & $6218.7 \AA$ & $6219.80 \AA$ & $6242.01 \AA$ & $6258.00 \AA$ & $\mathrm{Y}$ & Y & $\mathrm{Fe} I$ \\
\hline $6240.6462 \AA$ & $6240.40 \AA$ & $6240.90 \AA$ & $6267.01 \mathrm{~A}$ & $6274.00 \AA$ & $\mathrm{Y}$ & Y & $\mathrm{Fe} I$ \\
\hline $6246.3180 \AA$ & $6245.90 \AA$ & $6246.70 \AA$ & $6314.01 \AA$ & $6316.50 \AA$ & $\mathrm{Y}$ & Y & $\mathrm{Fe} \mathrm{I}$ \\
\hline $6252.5549 \AA$ & $6252.20 \AA$ & $6252.90 \AA$ & $6321.51 \AA$ & $6323.50 \AA$ & $\mathrm{Y}$ & Y & $\mathrm{Fe} \mathrm{I}$ \\
\hline $6270.2234 \AA$ & $6270.05 \AA$ & $6270.40 \AA$ & $6334.01 \AA$ & $6338.00 \AA$ & Y & Y & Fe I \\
\hline $6315.8110 \AA$ & $6315.60 \AA$ & $6316.00 \AA$ & $6428.01 \AA$ & $6436.00 \AA$ & $\mathrm{Y}$ & Y & $\mathrm{Fe} \mathrm{I}$ \\
\hline $6322.6850 \AA$ & $6322.40 \AA$ & $6323.00 \AA$ & $6436.01 \AA$ & $6443.00 \AA$ & $\mathrm{Y}$ & Y & $\mathrm{Fe} \mathrm{I}$ \\
\hline $6336.8234 \AA$ & $6336.52 \AA$ & $6337.12 \AA$ & $6455.01 \AA$ & $6457.00 \AA$ & $\mathrm{Y}$ & Y & $\mathrm{Fe} I$ \\
\hline $6481.8698 \AA$ & $6481.60 \AA$ & $6482.10 \AA$ & $6698.01 \AA$ & $6700.00 \AA$ & $\mathrm{Y}$ & $\mathrm{Y}$ & $\mathrm{Fe} \mathrm{I}$ \\
\hline
\end{tabular}

Notes. The segment mask is the range synthesised by SME and the line mask is the range used by SME when calculating the $\chi^{2}$ sum, as described in Sect. 3.3.1. The quality is the estimated quality of the atomic data and blending, as described in Sect. 3.3.2. 
Table B.1. continued.

\begin{tabular}{|c|c|c|c|c|c|c|c|}
\hline \multirow[t]{2}{*}{ Line centre } & \multicolumn{2}{|l|}{ Line mask } & \multicolumn{2}{|l|}{ Segment } & \multirow{2}{*}{$\begin{array}{l}\log g f \\
\text { quality flag }\end{array}$} & \multirow{2}{*}{$\begin{array}{l}\text { Blend } \\
\text { quality flag }\end{array}$} & \multirow[t]{2}{*}{ Species } \\
\hline & Start & End & Start & End & & & \\
\hline $4993.3502 \AA$ & $4993.05 \AA$ & $4993.65 \AA$ & $4990.01 \AA$ & $4998.00 \AA$ & $\mathrm{U}$ & $\mathrm{Y}$ & $\mathrm{Fe}$ II \\
\hline $5197.5675 \AA$ & $5197.27 \AA$ & $5197.87 \AA$ & $5213.51 \AA$ & $5221.00 \AA$ & $\mathrm{Y}$ & $\mathrm{U}$ & $\mathrm{Fe}$ II \\
\hline $5316.6087 \AA$ & $5316.30 \AA$ & $5316.70 \AA$ & $5310.01 \AA$ & $5321.00 \AA$ & $\mathrm{Y}$ & $\mathrm{Y}$ & $\mathrm{Fe}$ II \\
\hline $5316.7812 \AA$ & $5316.70 \AA$ & $5317.00 \AA$ & $5321.01 \AA$ & $5331.00 \AA$ & $\mathrm{Y}$ & $\mathrm{Y}$ & $\mathrm{Fe}$ II \\
\hline $5325.5523 \AA$ & $5325.40 \AA$ & $5325.75 \AA$ & $5378.01 \AA$ & $5390.00 \AA$ & $\mathrm{Y}$ & $\mathrm{U}$ & $\mathrm{Fe}$ II \\
\hline $5414.0698 \AA$ & $5413.88 \AA$ & $5414.30 \AA$ & $5422.01 \AA$ & $5427.00 \AA$ & $\mathrm{Y}$ & $\mathrm{U}$ & $\mathrm{Fe}$ II \\
\hline $5425.2485 \AA$ & $5424.98 \AA$ & $5425.50 \AA$ & $5431.01 \AA$ & $5438.00 \AA$ & $\mathrm{Y}$ & $\mathrm{U}$ & $\mathrm{Fe}$ II \\
\hline $5991.3709 \AA$ & $5991.25 \AA$ & $5991.47 \AA$ & $6118.01 \AA$ & $6124.00 \AA$ & $\mathrm{U}$ & $\mathrm{Y}$ & $\mathrm{Fe}$ II \\
\hline $6149.2459 \AA$ & $6149.10 \AA$ & $6149.40 \AA$ & $6160.01 \AA$ & $6171.00 \AA$ & $\mathrm{U}$ & $\mathrm{Y}$ & $\mathrm{Fe}$ II \\
\hline $6432.6757 \AA$ & $6432.55 \AA$ & $6432.81 \AA$ & $6481.01 \AA$ & $6482.50 \AA$ & $\mathrm{Y}$ & $\mathrm{Y}$ & $\mathrm{Fe}$ II \\
\hline $5261.7040 \AA$ & $5261.45 \AA$ & $5261.90 \AA$ & $5310.01 \AA$ & $5321.00 \AA$ & $\mathrm{Y}$ & $\mathrm{U}$ & $\mathrm{Ca}$ \\
\hline $5581.9650 \AA$ & $5581.40 \AA$ & $5582.40 \AA$ & $5584.01 \AA$ & $5591.00 \AA$ & $\mathrm{Y}$ & $\mathrm{U}$ & $\mathrm{Ca}$ \\
\hline $5588.7490 \AA$ & $5588.30 \AA$ & $5589.10 \AA$ & $5676.01 \AA$ & $5683.00 \AA$ & $\mathrm{Y}$ & $\mathrm{Y}$ & $\mathrm{Ca}$ \\
\hline $5867.5620 \AA$ & $5867.40 \AA$ & $5867.70 \AA$ & $5956.01 \AA$ & $5957.50 \AA$ & $\mathrm{Y}$ & $\mathrm{Y}$ & $\mathrm{Ca}$ \\
\hline $6122.2170 \AA$ & $6121.00 \AA$ & $6123.50 \AA$ & $6160.01 \AA$ & $6171.00 \AA$ & $\mathrm{Y}$ & $\mathrm{U}$ & $\mathrm{Ca}$ \\
\hline $6162.1730 \AA$ & $6161.50 \AA$ & $6163.00 \AA$ & $6160.01 \AA$ & $6171.00 \AA$ & $\mathrm{Y}$ & $\mathrm{Y}$ & $\mathrm{Ca}$ \\
\hline $6166.4390 \AA$ & $6166.30 \AA$ & $6166.60 \AA$ & $6171.01 \AA$ & $6179.00 \AA$ & $\mathrm{Y}$ & $\mathrm{Y}$ & $\mathrm{Ca}$ \\
\hline $6169.0420 \AA$ & $6168.80 \AA$ & $6169.18 \AA$ & $6198.01 \AA$ & $6202.00 \AA$ & $\mathrm{Y}$ & $\mathrm{Y}$ & $\mathrm{Ca}$ \\
\hline $6169.5630 \AA$ & $6169.20 \AA$ & $6169.90 \AA$ & $6217.01 \AA$ & $6223.00 \AA$ & $\mathrm{Y}$ & $\mathrm{Y}$ & $\mathrm{Ca}$ \\
\hline $6439.0750 \AA$ & $6438.00 \AA$ & $6440.30 \AA$ & $6498.01 \AA$ & $6501.00 \AA$ & $\mathrm{Y}$ & $\mathrm{U}$ & $\mathrm{Ca}$ \\
\hline $6455.5980 \AA$ & $6455.40 \AA$ & $6455.80 \AA$ & $6626.51 \AA$ & $6628.50 \AA$ & $\mathrm{Y}$ & $\mathrm{Y}$ & $\mathrm{Ca}$ \\
\hline $6499.6500 \AA$ & $6499.30 \AA$ & $6500.00 \AA$ & $6747.01 \AA$ & $6752.00 \AA$ & Y & $\mathrm{Y}$ & $\mathrm{Ca}$ \\
\hline $5167.3216 \AA$ & $5165.70 \AA$ & $5169.50 \AA$ & $5160.01 \AA$ & $5180.00 \AA$ & $\mathrm{Y}$ & $\mathrm{U}$ & $\mathrm{Mg}$ \\
\hline $5183.6042 \AA$ & $5181.00 \AA$ & $5187.00 \AA$ & $5190.01 \AA$ & $5201.00 \AA$ & $\mathrm{Y}$ & $\mathrm{Y}$ & $\mathrm{Mg}$ \\
\hline $5528.4047 \AA$ & $5527.60 \AA$ & $5528.70 \AA$ & $5541.01 \AA$ & $5547.00 \AA$ & $\mathrm{U}$ & $\mathrm{Y}$ & $\mathrm{Mg}$ \\
\hline
\end{tabular}

\section{Appendix C: Cores of strong lines}

Even with the NLTE grids described in Sect. 3.3.4, SME cannot reliably model the NLTE-dominated cores of strong lines. Hence, the pipeline automatically removes from the line mask every pixel within $4 \AA$ of the line centre where the modelled flux falls below a threshold. The threshold intensity is denoted $c_{\text {min }}$ and is given by

$c_{\min } \equiv\left\{\begin{array}{cr}0.60, & -1<[\mathrm{Fe} / \mathrm{H}] \\ 0.65, & -2<[\mathrm{Fe} / \mathrm{H}]<-1 \\ 0.72, & {[\mathrm{Fe} / \mathrm{H}]<-2}\end{array}\right.$.

\section{Appendix D: Continuum mask}

The segment and line masks are defined ahead of time. Instead, the continuum masks are defined algorithmically for each segment when the pipeline is run. The algorithm attempts to find continuum points by avoiding those points (based on the line list) that are known to be parts of lines, or (based on the observed spectrum) that appear to be parts of either telluric lines or stellar lines missing from the line list. To do this we define the parameter $c_{\text {frac }}$ that is used to determine the minimum observed intensity a pixel must have to be included in the continuum mask. We also define the parameter $c_{\mathrm{wt}}$ that is used to determine the minimum model intensity a pixel must have to be included in the mask. Finally, we define the parameter $e_{\text {frac }}$ that defines the maximum observed intensity a pixel must have to be included in the mask. This is intended to remove atmospheric emission lines.
The algorithm is defined as follows: First a preliminary normalisation is done of each segment by robustly fitting a straight line to the highest points in the observed spectrum, using the robust_poly_fit routine from the IDLAstro library (Landsman 1993). Then any pixel outside of the line mask will be assigned to the continuum mask if all of the following are true:

- The observed intensity is among the $c_{\text {frac }}+e_{\text {frac }}$ highest;

- The model intensity is among the $c_{\mathrm{wt}} \cdot c_{\mathrm{frac}}+e_{\mathrm{frac}}$ highest;

- The observed intensity is not among the $e_{\text {frac }}$ highest.

If this results in fewer than ten pixels being assigned to the continuum mask in the left one-third or the right one-third of the segment, $c_{\text {frac }}$ is increased enough for that third to include ten pixels.

We note that the choice to compare model pixels based to the product $c_{\mathrm{wt}} \cdot c_{\mathrm{frac}}$ is purely one of convenience. We could just as easily have defined one parameter $c_{\text {frac,model }}$ and required that the model intensity lie among the $c_{\text {frac,model }}+e_{\text {frac }}$ brightest. The parameters were defined in this way because a user who wants to increase or decrease the strictness of the algorithm would generally want to increase $c_{\text {frac }}$ and $c_{\text {frac,model }}$ by the same proportion. This way, it is only necessary to change one value to do that.

Empirically, the values producing the best results have been found to be in the vicinity of $c_{\mathrm{frac}}=0.2, e_{\mathrm{frac}}=0.005$ and $c_{\mathrm{wt}}=3.0$.

\section{Appendix E: Turbulence parameters}

Instead of explicitly stating a starting guess for $v_{\text {mic }}$, we estimated this from $T_{\text {eff }}$ using a relation that was derived empirically within GES. While we do not fit $v_{\mathrm{mac}}$, a fixed value for this is also 
Table E.1. Parameters determining $v_{\text {mic }}^{\text {start }}$ and $v_{\mathrm{mac}}$.

\begin{tabular}{lll}
\hline \hline Parameter & Default & Special \\
\hline$T_{0} / \mathrm{K}$ & 5500 & 5500 \\
$a_{i, 1}$ & 1.1 & 1.1 \\
$a_{a, 1}$ & 1.5 & 1.5 \\
$a_{i, 2}$ & $1.6 \times 10^{-4}$ & $10^{-4}$ \\
$a_{i, 3}$ & 0 & $4 \times 10^{-7}$ \\
$b_{a, 1}$ & $0.2 \times 10^{-3}$ & 0 \\
$c_{a, 1}$ & 0 & $10^{-6}$ \\
\hline
\end{tabular}

calculated empirically:

$v_{\text {mic }}^{\text {start }}=a_{i, 1}+a_{i, 2}\left(T_{\text {eff }}-T_{0}\right)+a_{i, 3}\left(T_{\text {eff }}-T_{0}\right)^{2}$,

$v_{\mathrm{mac}}=3\left(a_{a, 1}+b_{a, 1}\left(T_{\mathrm{eff}}-T_{0}\right)+c_{a, 1}\left(T_{\mathrm{eff}}-T_{0}\right)^{2}\right)$.

As long as $T_{\text {eff }}>5500 \mathrm{~K}$ and $\log g>4.2$, a set of default values of the parameters is used. Otherwise we use a special set of values. We show the values in Table E.1.

\section{Appendix F: Estimating systematic offsets}

We assume that we have $N$ benchmark stars, which we index with $i$. For each star we have $n_{i}$ benchmark spectra, which we index with $k$. For each benchmark star and stellar parameter, there is some correct value $p_{i}^{\text {true }}$. Let $p_{i}^{\text {fund }}$ be the estimate of $p_{i}^{\text {true }}$ given by fundamental relations. Let $p_{i, k}$ be the estimate of $p_{i}^{\text {true }}$ given by analysing spectrum $k$ of star $i$ with the pipeline.

We assume that the estimates returned by fundamental relations can be described as the sum of the true value and one error term:

$p_{i, \text { fund }}=p_{i}^{\text {true }}+e_{i}^{\text {fund }}$

The term $e_{i}^{\text {fund }}$ is assumed to differ from star to star following a normal distribution with mean 0 and standard deviation $\sigma_{i}^{\text {fund }}$. We take the uncertainties stated in Heiter et al. (2015b) as our estimates of $\sigma_{i}^{\text {fund }}$. We assume that the estimates returned by the pipeline can be described as the sum of the true value and three error terms:

$p_{i, k}=p_{i}^{\text {true }}+e^{\text {pipe }}+e_{i}+e_{i, k}$.

The term $e^{\text {pipe }}$ represents the error due to inherent flaws and limitations in the pipeline. This could come from lines that are incorrectly described in the line list, or that are missing altogether. While this term is parameter-dependent, we assume that it can be treated as constant within some volume of parameter space. The term $e_{i}$ represents the error due to how the pipeline is affected by the peculiarities unique to each star. This could come from blends due to unusual abundances, from difficulties in observing the star that are constant over time, and the like. It is assumed to be constant for all spectra of a particular star, but to vary from star to star following a normal distribution of mean 0 and standard deviation $\sigma^{\text {star }}$. The term $e_{i, k}$ represents the error due to how the pipeline is affected by the peculiarities of each spectrum. This could come from Poissonian noise, from observational conditions specific to the observation night, and the like. It is assumed to vary from spectrum to spectrum following a normal distribution of mean 0 and standard deviation $\sigma^{\text {spec }}$.
Given these assumptions, the likelihood for a series of estimates $\left\{p_{i, k}\right\}$ is given by

$$
\begin{aligned}
& P\left(\left\{p_{i, k}\right\} \mid\left\{p_{i}^{\text {fund }}\right\}, e^{\text {pipe }}, \sigma^{\text {spec }}, \sigma^{\text {star }},\left\{\sigma_{i}^{\text {fund }}\right\}\right) \\
& \quad=\pi^{N / 2} \prod_{i=0}^{N} \frac{1}{D_{i} H_{i} \sqrt{A_{i}+E_{i}}} \exp \left(\frac{\left(B_{i}+F_{i}\right)^{2}}{A_{i}+E_{i}}-\left(C_{i}+G_{i}\right)\right),
\end{aligned}
$$

where we have introduced the shorthand

$$
\begin{aligned}
& A_{i} \equiv \frac{n_{i}}{2\left(\sigma_{i}^{\text {sum }}\right)^{2}} \text {, } \\
& B_{i} \equiv \frac{n_{i} e^{\mathrm{pipe}}-\sum_{k=0}^{n_{i}} p_{i, k}}{2\left(\sigma_{i}^{\mathrm{sum}}\right)^{2}} \\
& C_{i} \equiv \frac{\sum_{k=0}^{n_{i}}\left(e^{\mathrm{pipe}}-p_{i, k}\right)^{2}-\left(\frac{\sigma_{\text {star }}^{\text {sum }}}{\sigma_{i}^{2}}\right)^{2}\left(\sum_{k=0}^{n_{i}}\left(e^{\mathrm{pipe}}-p_{i, k}\right)\right)^{2}}{2\left(\sigma^{\text {spec }}\right)^{2}}, \\
& D_{i} \equiv(2 \pi)^{n_{i} / 2}\left(\sigma^{\mathrm{spec}}\right)^{n_{i}-1} \sigma_{i}^{\mathrm{sum}} \text {, } \\
& E_{i} \equiv \frac{1}{2\left(\sigma_{i}^{\text {fund }}\right)^{2}} \text {, } \\
& F_{i} \equiv-\frac{p_{i}^{\text {fund }}}{2\left(\sigma_{i}^{\text {fund }}\right)^{2}}, \\
& G_{i} \equiv \frac{\left(p_{i}^{\text {fund }}\right)^{2}}{2\left(\sigma_{i}^{\text {fund }}\right)^{2}}, \\
& H_{i} \equiv \sqrt{2 \pi} \sigma_{i}^{\text {fund }} \text {. }
\end{aligned}
$$

Given a sequence of estimates $\left\{p_{i, k}\right\}$, a sequence of fundamental estimates $\left\{p_{i}^{\text {fund }}\right\}$, and a series of uncertainties in the fundamental parameters $\left\{\sigma_{i}^{\text {fund }}\right\}$, we can then estimate $e^{\text {pipe }}, \sigma^{\text {star }}$, and $\sigma^{\text {spec }}$.

We use Goodman and Weare's Affine Invariant Markov chain Monte Carlo Ensemble Sampler to estimate the distribution $P$ (Foreman-Mackey et al. 2013). We then use the median of each error parameter as our best estimate of that parameter. We use the 68th percentile as our estimate of the uncertainty in the error parameter.

The resulting estimates of $e^{\text {pipe }}$ are shown in Table 2. Estimates of all three parameters are shown for $T_{\text {eff }}$ in Table F.1, for $\log g$ in Table F.2, and for $[\mathrm{Fe} / \mathrm{H}]$ in Table F.3.

While the parameters describing the scatter are reassuringly fairly small, their relative sizes are somewhat discouraging. How well we can determine $e^{\text {pipe }}$ is limited by $\sigma^{\text {spec }}$ and $\sigma^{\text {star }}$, and by $N$ and $n_{i}$. If it were the case that $\sigma^{\text {spec }}=\sigma^{\text {star }}=0$, then a single spectrum of a single benchmark star would be enough to determine $e^{\text {pipe }}$ exactly. If it were the case that $\sigma^{\text {spec }} \gg \sigma^{\text {star }}$, then spectrum-to-spectrum scatter would give the main contribution to the error in $e^{\text {pipe }}$, which could be dealt with by taking more spectra of each benchmark star. Unfortunately, to within a factor of a few $\sigma^{\text {spec }} \approx \sigma^{\text {star }}$, which means that to determine $e^{\text {pipe }}$ much more accurately, we would need to increase the sample of benchmark stars, which is non-trivial. For our fairly rough categorisation of stars into four types, and our assumption that $e^{\text {pipe }}$ is approximately constant, the number of stars currently available is sufficient. However, if we wanted to estimate the offset over smaller regions of parameter space, either by dividing the benchmark sample into more types of benchmark stars or by replacing the constant $e^{\text {pipe }}$ with some function of $T_{\mathrm{eff}}, \log g$, and $[\mathrm{Fe} / \mathrm{H}]$, we might not have enough benchmark stars. 
Table F.1. Estimated error parameters for $T_{\text {eff. }}$.

\begin{tabular}{llll}
\hline \hline Star type & $e^{\mathrm{pipe}} / \mathrm{K}$ & $\sigma^{\text {star }} / \mathrm{K}$ & $\sigma^{\text {spec }} / \mathrm{K}$ \\
\hline The Sun & $-25 \pm 4$ & $\ldots$ & 10 \\
Solar-type & $-30 \pm 40$ & $80 \pm 40$ & $20 \pm 40$ \\
F dwarfs & $50 \pm 40$ & $190 \pm 40$ & $20 \pm 40$ \\
FGK subg. & $0 \pm 20$ & $50 \pm 20$ & $60 \pm 40$ \\
Red giants & $50 \pm 20$ & $30 \pm 20$ & $70 \pm 20$ \\
\hline
\end{tabular}

Notes. We show the best estimates of the three parameters we used in modelling the behaviour of the pipeline. We also show the offset and spectrum-to-spectrum scatter specific to the Sun.

Table F.2. Estimated error parameters for $\log g$.

\begin{tabular}{llll}
\hline \hline Star type & $e^{\text {pipe }}$ & $\sigma^{\text {star }}$ & $\sigma^{\text {spec }}$ \\
\hline The Sun & $-0.070 \pm 0.004$ & $\ldots$ & 0.01 \\
Solar-type & $-0.04 \pm 0.03$ & $0.07 \pm 0.03$ & $0.02 \pm 0.04$ \\
F dwarfs & $0.08 \pm 0.05$ & $0.13 \pm 0.08$ & $0.08 \pm 0.06$ \\
FGK subg. & $0.02 \pm 0.03$ & $0.11 \pm 0.03$ & $0.15 \pm 0.03$ \\
Red giants & $0.15 \pm 0.01$ & $0.14 \pm 0.02$ & $0.12 \pm 0.02$ \\
\hline
\end{tabular}

Notes. We show the best estimates of the three parameters we used in modelling the behaviour of the pipeline. We also show the offset and spectrum-to-spectrum scatter specific to the Sun.

Table F.3. Estimated error parameters for $[\mathrm{Fe} / \mathrm{H}]$.

\begin{tabular}{llll}
\hline \hline Star type & $e^{\text {pipe }}$ & $\sigma^{\text {star }}$ & $\sigma^{\text {spec }}$ \\
\hline The Sun & $0.008 \pm 0.003$ & $\ldots$ & 0.008 \\
Solar-type & $0.003 \pm 0.004$ & $0.020 \pm 0.002$ & $0.016 \pm 0.002$ \\
F dwarfs & $0.019 \pm 0.008$ & $0.080 \pm 0.008$ & $0.034 \pm 0.008$ \\
FGK subg. & $-0.03 \pm 0.02$ & $0.05 \pm 0.02$ & $0.06 \pm 0.02$ \\
Red giants & $-0.07 \pm 0.05$ & $0.05 \pm 0.05$ & $0.06 \pm 0.05$ \\
\hline
\end{tabular}

Notes. We show the best estimates of the three parameters we used in modelling the behaviour of the pipeline. We also show the offset and spectrum-to-spectrum scatter specific to the Sun.

\section{Appendix G: M 67 spectra}

We show the full list of objects in M 67 for which we have spectra in Table G.1.
Table G.1. Members of the M 67 cluster for which we have spectra.

\begin{tabular}{|c|c|c|}
\hline Right ascension & Declination & Classification \\
\hline 08:50:49.95 & $+11: 49: 12.6$ & Variable \\
\hline $08: 51: 12.68$ & $+11: 52: 42.2$ & Red giant \\
\hline $08: 51: 17.11$ & $+11: 48: 16.0$ & Red giant \\
\hline $08: 51: 17.48$ & $+11: 45: 22.5$ & Red giant \\
\hline 08:51:19.89 & $+11: 47: 00.1$ & Blue straggler \\
\hline $08: 51: 22.83$ & $+11: 48: 01.5$ & Red giant \\
\hline $08: 51: 28.98$ & $+11: 50: 33.0$ & Red giant \\
\hline $08: 51: 29.34$ & $+11: 45: 27.4$ & \\
\hline $08: 51: 32.39$ & $+11: 48: 01.0$ & \\
\hline $08: 51: 35.39$ & $+11: 57: 56.2$ & Variable \\
\hline $08: 51: 36.00$ & $+11: 46: 33.3$ & Binary \\
\hline $08: 51: 39.22$ & $+11: 50: 03.7$ & \\
\hline $08: 51: 39.37$ & $+11: 51: 45.5$ & Red giant \\
\hline $08: 51: 42.33$ & $+11: 50: 07.5$ & Red giant \\
\hline $08: 51: 42.35$ & $+11: 51: 22.9$ & Red giant \\
\hline 08:51:43.99 & $+11: 46: 24.3$ & \\
\hline $08: 51: 44.73$ & $+11: 46: 45.8$ & Red giant \\
\hline 08:50:51.82 & $+11: 56: 55.9$ & Binary \\
\hline 08:50:56.00 & $+11: 53: 51.9$ & \\
\hline 08:50:58.91 & $+11: 48: 19.2$ & \\
\hline 08:51:00.17 & $+11: 54: 32.1$ & Variable \\
\hline $08: 51: 00.80$ & $+11: 48: 52.7$ & YBP-1194 \\
\hline $08: 51: 03.25$ & $+11: 45: 47.3$ & Variable \\
\hline $08: 51: 05.24$ & $+11: 49: 34.0$ & \\
\hline 08:51:08.38 & $+11: 47: 12.1$ & Red giant \\
\hline 08:51:09.69 & $+11: 59: 09.6$ & Binary \\
\hline $08: 51: 12.67$ & $+11: 50: 34.5$ & \\
\hline $08: 51: 17.99$ & $+11: 45: 54.1$ & Binary \\
\hline $08: 51: 18.54$ & $+11: 49: 21.4$ & Variable \\
\hline 08:51:18.68 & $+11: 47: 02.6$ & Variable \\
\hline $08: 51: 19.01$ & $+11: 50: 05.6$ & Variable \\
\hline $08: 51: 20.12$ & $+11: 46: 41.7$ & Variable \\
\hline $08: 51: 22.91$ & $+11: 48: 49.3$ & Binary \\
\hline $08: 51: 29.40$ & $+11: 54: 13.9$ & Binary \\
\hline $08: 51: 30.45$ & $+11: 48: 58.2$ & Red giant \\
\hline $08: 51: 33.22$ & $+11: 48: 51.3$ & Variable \\
\hline $08: 51: 35.77$ & $+11: 53: 34.7$ & Red giant \\
\hline $08: 51: 37.40$ & $+11: 50: 05.2$ & Variable \\
\hline 08:51:40.81 & $+11: 49: 05.5$ & \\
\hline $08: 51: 41.22$ & $+11: 54: 29.0$ & Variable \\
\hline $08: 51: 45.07$ & $+11: 47: 45.9$ & Red giant \\
\hline $08: 51: 49.95$ & $+11: 49: 31.1$ & Variable \\
\hline
\end{tabular}

